\title{
The Generalized Viscosity Implicit Rules of Asymptotically Nonexpansive Mappings in Hilbert Spaces
}

\author{
Sang B Mendy, John T Mendy*® , Alieu Jobe \\ University of the Gambia, Brikama Campus, Gambia \\ sangbm1@gmail.com,jt.mendy@utg.edu.gm, alieueejobe@gmail.com \\ *Correspondence: jt.mendy@utg.edu.gm
}

\begin{abstract}
The generalized viscosity implicit rules of nonexpansive asymptotically mappings in Hilbert spaces are considered. The strong convergence theorems of the rules are proved under certain assumptions imposed on the sequences of parameters. An application of it in the convex minimization problem is considered. The results presented in this paper improve and extend some recent corresponding results in the literature.
\end{abstract}

\section{Background}

Let $\mathcal{H}$ be a real Hilbert space and $\mathcal{M}$ be a nonempty closed convex subset of $\mathcal{H}, \mathcal{T}: \mathcal{M} \rightarrow \mathcal{M}$ be a nonexpansive mapping with a nonempty fixed point set $\mathrm{F}(\mathcal{T})$

The following iteration method is known as the viscosity approximation method: for arbitrarily chosen $u_{0} \in \mathcal{M}$

$$
u_{n+1}=\alpha_{n} \psi\left(u_{n}\right)+\left(1-\alpha_{n}\right) \mathcal{T} u_{n}, n \geq 0,
$$

where $\psi: \mathcal{M} \rightarrow \mathcal{M}$ is a contraction and $\left\{\alpha_{n}\right\}$ is a sequence in $(0,1)$. Under some certain conditions, the sequence $\left\{u_{n}\right\}$ converges strongly to a point $z \in F(\mathcal{T})$ which solves the variational inequality $(V I)$

$$
\langle(I-\psi) z, u-z\rangle \geq 0, u \in F(\mathcal{T})
$$

where $/$ is the identity of $\mathcal{H}$. Many authors studied iterative sequence for the implicit midpoint rule because of it's significant for solving ordinary differential equations; see [?]- [12], John T [9], [7] and the references therein. Recently, Xu et al [3] proposed the following viscosity implicit midpoint rule (VIMR) for nonexpansive mappings:

$$
u_{n+1}=\alpha_{n} \psi\left(u_{n}\right)+\left(1-\alpha_{n}\right) \mathcal{T}\left(\frac{u_{n}+u_{n+1}}{2}\right), n \geq 0,
$$

Received: 23 Aug 2021.

Key words and phrases. viscosity; Hilbert space; convex minimization; asymptotically nonexpansive mapping; variational inequality; fixed point. 
In 2015, Ke and Ma [4] proposed the generalized viscosity implicit rules of nonexpansive mappings in Hilbert spaces as follows:

$$
u_{n+1}=\alpha_{n} \psi\left(u_{n}\right)+\left(1-\alpha_{n}\right) \mathcal{T}\left(s_{n} u_{n}+\left(1-s_{n}\right) u_{n+1}\right), n \geq 0,
$$

and

$$
u_{n+1}=\alpha_{n} u_{n}+\beta_{n} \psi\left(u_{n}\right)+\gamma_{n} \mathcal{T}\left(s_{n} u_{n}+\left(1-s_{n}\right) u_{n+1}\right), n \geq 0,
$$

They proved that the generalized viscosity implicit rules 1.4 and 1.5 converge strongly to a fixed point of $\mathcal{T}$ under certain assumptions, which also solved the $\mathrm{V} I(1.1)$.

In 2016, motivated by the work of Xu [3], Zhao et al [5] proposed the following implicit midpoint rule for asymptotically nonexpansive mappings:

$$
u_{n+1}=\alpha_{n} \psi\left(u_{n}\right)+\left(1-\alpha_{n}\right) \mathcal{T}^{n}\left(\frac{u_{n}+u_{n+1}}{2}\right), n \geq 0
$$

where $\mathcal{T}$ is an asymptotically nonexpansive mapping. They proved that the sequence $\left\{u_{n}\right\}$ converges strongly to a fixed point of $\mathcal{T}$, which, in addition, also solves the $\mathrm{V} I(1.1)$.

In 2017, He et la [14] studied the following iterative

$$
u_{n+1}=\alpha_{n} \psi\left(u_{n}\right)+\left(1-\alpha_{n}\right) \mathcal{T}^{n}\left(\beta_{n} u_{n}+\left(1-\beta_{n}\right) u_{n+1}\right), n \geq 0
$$

in the setting of a Hilbert space and proved that the sequence $\left\{u_{n}\right\}$ converges strongly to $u^{*}=$ $P_{F(\mathcal{T})} \psi\left(u^{*}\right)$ which is also the unique solution of the following $V I$

$$
\langle(I-\psi) u, v-u\rangle \geq 0, \forall v \in F(\mathcal{T})
$$

In this paper, we introduce and study the generalized viscosity implicit rules of asymptotically nonexpansive mappings in Hilbert spaces. More precisely, we consider the following implicit iterative algorithm:

$$
\begin{cases}u_{1} & \in \mathcal{M} \\ u_{n+1} & =\alpha_{n} u_{n}+\beta_{n} \psi\left(u_{n}\right)+\gamma_{n} \mathcal{T}^{n}\left(s_{n} u_{n}+\left(1-s_{n}\right) u_{n+1}\right) \forall n \in \mathbb{N}\end{cases}
$$

Under suitable conditions, we proved that the sequence $\left\{u_{n}\right\}$ converge strongly to a fixed point of the asymptotically nonexpansive mapping $\mathcal{T}$, which also solves the variational inequality

$$
\langle(I-\psi) u, p-u\rangle \geq 0 \quad p \in F(\mathcal{T})
$$

As applications, we apply our results to solve convexly constrained minimization problem. This way results in 1.5 are complemented, extended and generalized. 


\section{Preliminaries}

In the sequel, we always assume that $\mathcal{H}$ is a real Hilbert space and $\mathcal{M}$ is a nonempty, closed, and convex subset of $\mathcal{H}$. The nearest point projection from $\mathcal{H}$ onto $\mathcal{M}, P \mathcal{M}$, is defined by

$$
P_{\mathcal{M}}(u):=\arg \min _{z \in \mathcal{M}}\|u-z\|^{2}, u \in \mathcal{H} .
$$

Namely, $P_{\mathcal{M}}(u)$ is the only point in $\mathcal{M}$ that minimizes the objective $\|u-z\|$ over $z \in \mathcal{M}$. and $P_{\mathcal{M}}(u)$ is characterized as follows:

$$
P_{\mathcal{M}}(u) \in \mathcal{M} \text { and }\left\langle u-P_{\mathcal{M}}(u), z-P_{\mathcal{M}}(u)\right\rangle \leq 0 \text { for all } z \in \mathcal{M} \text {. }
$$

Definition 2.1. . A mapping $\mathcal{T}: \mathcal{M} \rightarrow \mathcal{M}$ is said to be:

a): $\alpha$-inverse strongly monotone if there exists $\alpha>0$ satisfying

$$
\langle u-v, \mathcal{T} u-\mathcal{T} v\rangle \geq \alpha\|A u-A v\|^{2} \quad \forall u, v \in \mathcal{M}
$$

b): $L$-Lipschitz continuous if there exists $L \geq 0$ satisfying

$$
\|\mathcal{T} u-\mathcal{T} v\| \leq L\|u-v\| \quad \forall u, v \in \mathcal{M}
$$

c): nonexpansive if

$$
\|\mathcal{T} u-\mathcal{T} v\| \leq\|u-v\| \quad \forall u, v \in \mathcal{M} ;
$$

d): asymptotically nonexpansive if there exists a sequence $\left\{k_{n}\right\} \subset[1, \infty)$ with $\lim _{n \rightarrow \infty} k_{n}=1$ such that

$$
\left\|\mathcal{T}^{n} u-\mathcal{T}^{n} v\right\| \leq k_{n}\|u-v\| \quad \forall u, v \in \mathcal{M} \quad \text { and } \quad \forall n \in \mathbb{N}
$$

e): contraction if there exists the contractive constant $\alpha \in[0,1)$ such that

$$
\|\mathcal{T} u-\mathcal{T} v\| \leq \alpha\|u-v\| \forall u, v \in \mathcal{M}
$$

Lemma 2.2. (The demiclosedness principle [10]). Let $\mathcal{H}$ be a Hilbert space, $\mathcal{M}$ be a nonempty closed convex subset of $\mathcal{H}$, and $\mathcal{T}: \mathcal{M} \rightarrow \mathcal{M}$ be a asymptotically nonexpansive mapping with $\operatorname{Fix}(\mathcal{T}) \neq \emptyset$. If $\left\{u_{n}\right\}$ is a sequence in $\mathcal{M}$ such that $\left\{u_{n}\right\}$ weakly converges to $u$ and $\left\{(I-\mathcal{T}) u_{n}\right\}$ converges strongly to 0 , then $u=\mathcal{T}(u)$

Lemma 2.3. Let $\mathcal{H}$ be a Hilbert space. Then for all $\theta, u, v \in \mathcal{H}$, the following inequality holds

$$
\|u-\theta\|^{2} \leq\|v-\theta\|^{2}+2\langle u-v, u-\theta\rangle
$$

Lemma 2.4. [11]). Assume that $\left\{\alpha_{n}\right\}$ is a sequence of nonnegative real numbers such that

$$
\alpha_{n+1} \leq\left(1-\lambda_{n}\right) \alpha_{n}+\delta_{n}
$$

for all $n \in \mathbb{N}$, where $\left\{\lambda_{n}\right\} \subseteq(0,1)$ and $\left\{\delta_{n}\right\} \subseteq \mathbb{R}$ are two sequences satisfying the following conditions: 
(i): $\sum_{n=1}^{\infty} \lambda_{n}=\infty$

(ii): $\lim \sup _{n \rightarrow \infty} \frac{\delta_{n}}{\lambda_{n}} \leq 0$ or $\sum_{n=1}^{\infty}\left|\delta_{n}\right|<\infty$

Then $\lim _{n \rightarrow \infty} \alpha_{n}=0$

Then the sequence $\left\{\alpha_{n}\right\}$ converges to 0 .

\section{Main Result}

We now prove the following new result.

Theorem 3.1. Let $\mathcal{M}$ be a nonempty closed convex subset a real Hilbert space $H, \mathcal{T}: \mathcal{M} \rightarrow$ $\mathcal{M}$ be asymptotically nonexpansive mappings with the same sequence $\left\{k_{n}\right\} \subseteq[1, \infty)$ such that $\lim _{n \rightarrow \infty} k_{n}=1, \operatorname{Fix}(\mathcal{T}) \neq \emptyset$ and $\psi: \mathcal{M} \rightarrow \mathcal{M}$ be a contraction mapping with the contractive constant $\alpha \in[0,1)$. Define a sequence $\left\{u_{n}\right\}$ in $\mathcal{M}$ as follows:

$$
\begin{cases}u_{1} & \in \mathcal{M} \\ u_{n+1} & =\alpha_{n} u_{n}+\beta_{n} \psi\left(u_{n}\right)+\gamma_{n} \mathcal{T}^{n}\left(s_{n} u_{n}+\left(1-s_{n}\right) u_{n+1}\right) \forall n \in \mathbb{N}\end{cases}
$$

where $\alpha_{n}, \beta_{n}, \gamma_{n}, s_{n} \in(0,1)$ satisfying the following conditions,

A1: $\alpha_{n}+\beta_{n}+\gamma_{n}=1$

A2: $\sum_{n=0}^{\infty} \alpha_{n}=\infty$

A3: $0<\epsilon \leq s_{n} \leq s_{n+1}<1$ for all $n \geq 0$

A4: $\lim _{n \rightarrow \infty} \gamma_{n}=1$ and $\lim _{n \rightarrow \infty} \alpha_{n}=\lim _{n \rightarrow \infty} \beta_{n}=\lim _{n \rightarrow \infty} s_{n}=0$

$$
\lim _{n \rightarrow \infty}\left\|u_{n}-\mathcal{T}^{n} u_{n}\right\|=0
$$

Then the sequence $\left\{u_{n}\right\}$ strongly converges to a common fixed point $q$ of $\mathcal{T}$, which is also the unique solution of the following variational inequality

$$
\langle(I-\psi) u, p-u\rangle \geq 0 \quad p \in F(\mathcal{T}) .
$$

We now show that algorithm 3.1 is well posed. Letting

$$
\begin{aligned}
B_{n}(u) & =\alpha_{n} u_{n}+\beta_{n} \psi\left(u_{n}\right)+\gamma_{n} \mathcal{T}^{n}\left(s_{n} u_{n}+\left(1-s_{n}\right) u_{n}\right) \\
\left\|B_{n}(u)-B_{n}(v)\right\| & =\left\|\gamma_{n} \mathcal{T}^{n}\left(s_{n} u_{n}+\left(1-s_{n}\right) u\right)-\gamma_{n} \mathcal{T}^{n}\left(s_{n} u_{n}+\left(1-s_{n}\right) v\right)\right\| \\
& =\left\|\gamma_{n} \mathcal{T}^{n}\left(1-s_{n}\right) u-\gamma_{n} \mathcal{T}^{n}\left(1-s_{n}\right) v\right\| \\
& \leq \gamma_{n} k_{n}\left(1-s_{n}\right)\|u-v\|
\end{aligned}
$$

Since $\lim _{n \rightarrow \infty} s_{n}=0, \lim _{n \rightarrow \infty} k_{n}=1, \lim _{n \rightarrow \infty} \gamma_{n}=1$ and $0<\epsilon \leq s_{n} \leq s_{n+1}<1$ for all $n>0$, we may assume that $\gamma_{n} k_{n}\left(1-s_{n}\right) \leq 1-\epsilon$ for all $n>0$. This implies that $B_{n}$ is a contraction for each 
n. Therefore there exists a unique fixed point for $B_{n}$ by Banach contraction principle, which also implies that (3.1) is well-defined.

We now show that the sequence $\left\{u_{n}\right\}$ is bounded.

Rewriting 3.1, we have

$$
u_{n+1}=\beta_{n} \psi\left(u_{n}\right)+\alpha_{n} u_{n}+\left(1-\beta_{n}\right) v_{n}
$$

where $v_{n}=\frac{\gamma_{n} \mathcal{T}^{n}\left(s_{n} u_{n}+\left(1-s_{n}\right) u_{n+1}\right)}{1-\beta_{n}}$

Remark 3.2. The real sequences that satisfies the above conditions are $\alpha_{n}=\frac{1}{n}, \beta_{n}=\frac{1}{n}$ and $\gamma_{n}=1-\frac{2}{n}$

Proof. Our prove are in six steps. First we prove that the sequence $\left\{u_{n}\right\}$ defined by 3.1 is bounded.

Step 1: Letting $p \in F i x(\mathcal{T})$, we have the following estimates

$$
\begin{aligned}
& \left\|u_{n+1}-p\right\|=\quad\left\|\beta_{n} \psi\left(u_{n}\right)+\alpha_{n} u_{n}+\left(1-\beta_{n}\right) v_{n}-p\right\| \\
& \leq \beta_{n}\left\|\psi\left(u_{n}\right)-\psi(p)\right\|+\beta_{n}\|\psi(p)-p\|+\alpha_{n}\left\|u_{n}-p\right\|+\left(1-\beta_{n}\right)\left\|v_{n}-p\right\| \\
& \leq\left(\alpha \beta_{n}+\alpha_{n}\right)\left\|u_{n}-p\right\|+\beta_{n}\|\psi(p)-p\|+\left(1-\beta_{n}\right)\left\|v_{n}-p\right\| \\
& \qquad \begin{aligned}
\left\|v_{n}-p\right\|= & \left\|\frac{\gamma_{n} \mathcal{T}^{n}\left(s_{n} u_{n}+\left(1-s_{n}\right) u_{n+1}\right)}{1-\beta_{n}}-p\right\| \\
& =\frac{\gamma_{n} \mathcal{T}^{n} s_{n}\left(u_{n}-p\right)}{1-\beta_{n}}+\frac{\gamma_{n} \mathcal{T}^{n}\left(1-s_{n}\right)\left(u_{n+1}-p\right)}{1-\beta_{n}} \| \\
& \leq \frac{\gamma_{n} k_{n} s_{n}}{1-\beta_{n}}\left\|u_{n}-p\right\|+\frac{\gamma_{n} k_{n}\left(1-s_{n}\right)}{1-\beta_{n}}\left\|u_{n+1}-p\right\|
\end{aligned}
\end{aligned}
$$

Putting 3.4 in 3.3, gives the following

$$
\begin{aligned}
\left\|u_{n+1}-p\right\| & \leq\left(\alpha \beta_{n}+\alpha_{n}\right)\left\|u_{n}-p\right\|+\beta_{n}\|\psi(p)-p\| \\
& +\gamma_{n} k_{n} s_{n}\left\|u_{n}-P\right\|+\gamma_{n} k_{n}\left(1-s_{n}\right)\left\|u_{n+1}-p\right\| \\
\left(1-\gamma_{n} k_{n}\left(1-s_{n}\right)\right)\left\|u_{n+1}-p\right\| & \leq\left(\alpha \beta_{n}+\alpha_{n}+\gamma_{n} k_{n} s_{n}\right)\left\|u_{n}-p\right\|+\beta_{n}\|\psi(p)-p\| \\
\left\|u_{n+1}-p\right\| & \leq \frac{\left(\alpha \beta_{n}+\alpha_{n}+\gamma_{n} k_{n} s_{n}\right)}{1-\gamma_{n} k_{n}\left(1-s_{n}\right)}\left\|u_{n}-p\right\| \\
& +\frac{\beta_{n}}{1-\gamma_{n} k_{n}\left(1-s_{n}\right)}\|\psi(p)-p\|
\end{aligned}
$$


Since $\gamma_{n}, s_{n} \in(0,1), 1-\gamma_{n} k_{n}\left(1-s_{n}\right)>0$ and $\lim _{n \rightarrow \infty} k_{n}=1$. From the condition $(A 1)$, we have

$$
\begin{aligned}
\left\|u_{n+1}-p\right\| & \leq 1-\frac{1-\alpha \beta_{n}-\alpha_{n}-\gamma_{n} k_{n}}{1-\gamma_{n} k_{n}\left(1-s_{n}\right)}\left\|u_{n}-p\right\| \\
& \left.+\frac{\beta_{n}}{1-\gamma_{n} k_{n}\left(1-s_{n}\right)}\|\psi(p)-p\|\right] \\
\left\|u_{n+1}-p\right\| & \leq 1-\frac{\beta_{n}(1-\alpha)}{1-\gamma_{n} k_{n}\left(1-s_{n}\right)}\left\|u_{n}-p\right\| \\
& \left.+\frac{\beta_{n}(1-\alpha)}{1-\gamma_{n} k_{n}\left(1-s_{n}\right)} \frac{1}{(1-\alpha)}\|\psi(p)-p\|\right] \\
\left\|u_{n+1}-p\right\| & \leq \max \left\{\left\|u_{n}-p\right\|, \frac{1}{(1-\alpha)}\|\psi(p)-p\|\right\}
\end{aligned}
$$

Therefore by mathematical induction, we have

$$
\left\|u_{n+1}-p\right\| \leq \max \left\{\left\|u_{0}-p\right\|, \frac{1}{(1-\alpha)}\|\psi(p)-p\|\right\}
$$

for all $n \geq N$. Therefore $\left\{u_{n}\right\}$ is bounded. Consequently,

$$
\left\{\psi\left(u_{n}\right)\right\} \text { and }\left\{v_{n}\right\}
$$

are also bounded.

Step 2: We now prove that the sequence $\left\{u_{n+1}\right\}$ converges to $\left\{u_{n}\right\}$ as $n \rightarrow \infty$. That is $\lim _{n \rightarrow \infty} \| u_{n+1}-$

$$
u_{n} \|=0
$$

$$
\begin{aligned}
\left\|u_{n+1}-u_{n}\right\|= & \left\|u_{n+1}-\mathcal{T}^{n} u_{n}+\mathcal{T}^{n} u_{n}-u_{n}\right\| \\
= & \left\|\beta_{n} \psi\left(u_{n}\right)+\alpha_{n} u_{n}+\left(1-\beta_{n}\right) v_{n}-\left(\beta_{n}+\alpha_{n}+\gamma_{n}\right) \mathcal{T}^{n}+\mathcal{T}^{n} u_{n}-u_{n}\right\| \\
\leq & \left\|\beta_{n} \psi\left(u_{n}\right)-\beta_{n} \mathcal{T}^{n} u_{n}\right\|+\left\|\alpha_{n} u_{n}-\alpha_{n} \mathcal{T}^{n} u_{n}\right\| \\
& +\left\|\left(1-\beta_{n}\right) v_{n}-\gamma_{n} \mathcal{T}^{n}+\mathcal{T}^{n} u_{n}-u_{n}\right\| \\
\leq & \beta_{n}\left\|\psi\left(u_{n}\right)-\mathcal{T}^{n} u_{n}\right\|+\alpha_{n}\left\|u_{n}-\mathcal{T}^{n} u_{n}\right\| \\
& +\left(1-\beta_{n}\right)\left\|v_{n}-\gamma_{n} \mathcal{T}^{n}\right\|+\left\|\mathcal{T}^{n} u_{n}-u_{n}\right\| \\
\left\|v_{n}-\gamma_{n} \mathcal{T}^{n} u_{n}\right\| & =\left\|\frac{\gamma_{n} s_{n}}{1-\beta_{n}} \mathcal{T}^{n} u_{n}+\frac{\gamma_{n}\left(1-s_{n}\right)}{1-\beta_{n}} \mathcal{T}^{n} u_{n+1}-\gamma_{n} \mathcal{T}^{n} u_{n}\right\| \\
\leq & \left\|\frac{\gamma_{n} s_{n}}{1-\beta_{n}}\right\| \mathcal{T}^{n} u_{n}-\mathcal{T}^{n} u_{n}\left\|+\frac{\gamma_{n}\left(1-s_{n}\right)}{1-\beta_{n}}\right\| \mathcal{T}^{n} u_{n+1}-\mathcal{T}^{n} u_{n} \| \\
\leq & \frac{\gamma_{n}\left(1-s_{n}\right) k_{n}}{1-\beta_{n}}\left\|u_{n+1}-u_{n}\right\|
\end{aligned}
$$


Now putting 3.6 in 3.5, we have the following

$$
\begin{aligned}
\left\|u_{n+1}-u_{n}\right\| \leq & \beta_{n}\left\|\psi\left(u_{n}\right)-\mathcal{T}^{n} u_{n}\right\|+\alpha_{n}\left\|u_{n}-\mathcal{T}^{n} u_{n}\right\| \\
& +\left(1-\beta_{n}\right)\left[\frac{\gamma_{n}\left(1-s_{n}\right) k_{n}}{1-\beta_{n}}\left\|u_{n+1}-u_{n}\right\|\right]+\left\|\mathcal{T}^{n} u_{n}-u_{n}\right\| \\
\leq & \beta_{n}\left\|\psi\left(u_{n}\right)-\mathcal{T}^{n} u_{n}\right\|+\alpha_{n}\left\|u_{n}-\mathcal{T}^{n} u_{n}\right\| \\
& \left.+\gamma_{n}\left(1-s_{n}\right) k_{n}\left\|u_{n+1}-u_{n}\right\|\right]+\left\|\mathcal{T}^{n} u_{n}-u_{n}\right\| \\
\leq & \beta_{n}\left\|\psi\left(u_{n}\right)-\mathcal{T}^{n} u_{n}\right\|+\left(\alpha_{n}+1\right)\left\|u_{n}-\mathcal{T}^{n} u_{n}\right\| \\
& +\gamma_{n}\left(1-s_{n}\right) k_{n}\left\|u_{n+1}-u_{n}\right\| \\
{\left[1-\gamma_{n}\left(1-s_{n}\right) k_{n}\right]\left\|u_{n+1}-u_{n}\right\| \leq } & \beta_{n}\left\|\psi\left(u_{n}\right)-\mathcal{T}^{n} u_{n}\right\|+\left(\alpha_{n}+1\right)\left\|u_{n}-\mathcal{T}^{n} u_{n}\right\| \\
\left\|u_{n+1}-u_{n}\right\| \leq & \frac{\beta_{n}}{1-\gamma_{n}\left(1-s_{n}\right) k_{n}}\left\|\psi\left(u_{n}\right)-\mathcal{T}^{n} u_{n}\right\| \\
& +\frac{\left(\alpha_{n}+1\right)}{1-\gamma_{n}\left(1-s_{n}\right) k_{n}}\left\|u_{n}-\mathcal{T}^{n} u_{n}\right\|
\end{aligned}
$$

Let $M:>\max \left\{\left\|\psi\left(u_{n}\right)-\mathcal{T}^{n} u_{n}\right\|\right\}$, then we have

$$
\begin{gathered}
\left\|u_{n+1}-u_{n}\right\| \leq \frac{\beta_{n} M}{1-\gamma_{n}\left(1-s_{n}\right) k_{n}}+\frac{\left(\alpha_{n}+1\right)}{1-\gamma_{n}\left(1-s_{n}\right) k_{n}}\left\|u_{n}-\mathcal{T}^{n} x_{n}\right\| \\
\left\|u_{n+1}-u_{n}\right\| \leq \frac{\beta_{n} M}{1-\gamma_{n}\left(1-s_{n}\right)\left(1+\epsilon \alpha_{n}\right)}+\frac{\left(\alpha_{n}+1\right)}{1-\gamma_{n}\left(1-s_{n}\right)\left(1+\epsilon \alpha_{n}\right)}\left\|u_{n}-\mathcal{T}^{n} u_{n}\right\|
\end{gathered}
$$

Since $\lim _{n \rightarrow \infty} \alpha_{n}=\lim _{n \rightarrow \infty} \beta_{n}=\lim _{n \rightarrow \infty}\left\|u_{n}-\mathcal{T}^{n} u_{n}\right\|=0$, we then conclude that $\lim _{n \rightarrow \infty} \| u_{n+1}-$ $u_{n} \|=0$

Step 3: Again we then show that $\lim _{n \rightarrow \infty}\left\|u_{n}-\mathcal{T}\left(u_{n}\right)\right\|=0$. Estimating as follows we have

$$
\begin{aligned}
\left\|u_{n}-\mathcal{T}^{n} u_{n}\right\| & =\left\|u_{n}-u_{n+1}+u_{n+1}-\mathcal{T}^{n} u_{n}\right\| \\
& \leq\left\|u_{n}-u_{n+1}\right\|+\left\|u_{n+1}-\mathcal{T}^{n} u_{n}\right\| \\
& \leq\left\|u_{n}-u_{n+1}\right\|+\left\|\beta_{n} \psi\left(u_{n}\right)+\alpha_{n} u_{n}+\left(1-\beta_{n}\right) v_{n}-\mathcal{T}^{n} u_{n}\right\| \\
& \leq\left\|u_{n}-u_{n+1}\right\|+\beta_{n}\left\|\psi\left(u_{n}\right)-\mathcal{T}^{n} u_{n}\right\|+\alpha_{n}\left\|u_{n}-\mathcal{T}^{n} u_{n}\right\|+\left(1-\beta_{n}\right)\left\|v_{n}-\gamma_{n} \mathcal{T}^{n} u_{n}\right\|
\end{aligned}
$$

$$
\begin{aligned}
\left\|v_{n}-\gamma_{n} \mathcal{T}^{n} u_{n}\right\| & =\left\|\frac{\gamma_{n} \mathcal{T}^{n}\left(s_{n} u_{n}+\left(1-s_{n}\right) u_{n+1}\right)}{1-\beta_{n}}-\gamma_{n} \mathcal{T}^{n} u_{n}\right\| \\
& \leq\left\|\frac{\gamma_{n} s_{n}}{1-\beta_{n}}\right\| \mathcal{T}^{n} u_{n}-\mathcal{T}^{n} u_{n}\left\|+\frac{\left(1-s_{n}\right) \gamma_{n}}{1-\beta_{n}}\right\| \mathcal{T}^{n} u_{n+1}-\mathcal{T}^{n} u_{n} \| \\
& \leq \frac{\left(1-s_{n}\right) \gamma_{n} k_{n}}{1-\beta_{n}}\left\|u_{n+1}-u_{n}\right\|
\end{aligned}
$$


Now substituting 3.8 into 3.7, gives the following estimation

$$
\begin{aligned}
\left\|u_{n}-\mathcal{T}^{n} u_{n}\right\| \leq & \left\|u_{n}-u_{n+1}\right\|+\beta_{n}\left\|\psi\left(u_{n}\right)-\mathcal{T}^{n} u_{n}\right\|+\alpha_{n}\left\|u_{n}-\mathcal{T}^{n} u_{n}\right\| \\
& +\left(1-\beta_{n}\right)\left(\frac{\left(1-s_{n}\right) \gamma_{n} k_{n}}{1-\beta_{n}}\left\|u_{n+1}-u_{n}\right\|\right) \\
\leq & \left(1+\left(1-s_{n}\right) \gamma_{n} k_{n}\right)\left\|u_{n}-u_{n+1}\right\|+\beta_{n}\left\|\psi\left(u_{n}\right)-\mathcal{T}^{n} u_{n}\right\|+\alpha_{n}\left\|u_{n}-\mathcal{T}^{n} u_{n}\right\| \\
\leq & \frac{\left(1+\left(1-s_{n}\right) \gamma_{n} k_{n}\right)}{1-\alpha_{n}}\left\|u_{n}-u_{n+1}\right\|+\frac{\beta_{n}}{1-\alpha_{n}}\left\|\psi\left(u_{n}\right)-\mathcal{T}^{n} u_{n}\right\| \\
\left\|u_{n}-\mathcal{T}^{n} u_{n}\right\| \leq & \frac{\left(1+\left(1-s_{n}\right) \gamma_{n} k_{n}\right)}{1-\alpha_{n}}\left\|u_{n+1}-x_{n}\right\|+\frac{\beta_{n} M}{1-\alpha_{n}}
\end{aligned}
$$

Therefore from 3.1 condition $A 4$, with $\lim _{n \rightarrow \infty}\left\|u_{n+1}-u_{n}\right\|=0$, we can conclude that

$$
\lim _{n \rightarrow \infty}\left\|u_{n}-\mathcal{T}^{n} u_{n}\right\|=0
$$

But we know that from the following fact

$$
\begin{aligned}
\lim _{n \rightarrow \infty}\left\|u_{n}-\mathcal{T}\left(u_{n}\right)\right\| & \leq \lim _{n \rightarrow \infty}\left\|u_{n}-\mathcal{T}^{n} u_{n}\right\|+\lim _{n \rightarrow \infty}\left\|\mathcal{T}^{n} u_{n}-\mathcal{T} x_{n}\right\| \\
& \leq \lim _{n \rightarrow \infty}\left\|u_{n}-\mathcal{T}^{n} u_{n}\right\|+\lim _{n \rightarrow \infty} k_{1}\left\|\mathcal{T}^{n-1} u_{n}-u_{n}\right\|
\end{aligned}
$$

Proving that $\lim _{n \rightarrow \infty}\left\|T^{n-1} u_{n}-u_{n}\right\|=0$, we have the following estimation

$$
\begin{aligned}
\left\|\mathcal{T}^{n-1}\left(u_{n}\right)-u_{n}\right\|= & \left\|u_{n}-\mathcal{T}^{n-1}\left(u_{n}\right)\right\| \\
= & \| \beta_{n-1} \psi\left(u_{n-1}\right)+\alpha_{n-1} u_{n-1}+\left(1-\beta_{n-1}\right) v_{n-1} \\
& -\left(\beta_{n-1}+\alpha_{n-1}+\gamma_{n-1}\right) \mathcal{T}^{n-1} u_{n} \| \\
= & \| \beta_{n-1} \psi\left(u_{n-1}\right)-\beta_{n-1} \mathcal{T}^{n-1} u_{n}+\alpha_{n-1} u_{n-1}-\alpha_{n-1} \mathcal{T}^{n-1} u_{n} \\
& +\left(1-\beta_{n-1}\right) v_{n-1}-\gamma_{n-1} \mathcal{T}^{n-1} u_{n} \| \\
\leq & \beta_{n-1}\left\|\psi\left(u_{n-1}\right)-\mathcal{T}^{n-1} u_{n}\right\|+\alpha_{n-1}\left\|u_{n-1}-\mathcal{T}^{n-1} u_{n}\right\| \\
& +\left(1-\beta_{n-1}\right)\left\|v_{n-1}-\gamma_{n-1} T^{n-1} x_{n}\right\| \\
\left\|v_{n-1}-\gamma_{n-1} \mathcal{T}^{n-1} u_{n}\right\| \leq & \left\|\frac{\gamma_{n-1} \mathcal{T}^{n-1}\left(s_{n-1} u_{n-1}+\left(1-s_{n-1}\right) u_{n}\right)}{1-\beta_{n-1}}-\gamma_{n-1} \mathcal{T}^{n-1} u_{n}\right\| \\
\leq & \frac{\gamma_{n-1} k_{n-1} s_{n-1}}{1-\beta_{n-1}}\left\|u_{n}-u_{n-1}\right\|
\end{aligned}
$$


combining 3.12 and 3.11 we have the following

$$
\begin{aligned}
\left\|\mathcal{T}^{n-1}\left(u_{n}\right)-u_{n}\right\| \leq & \beta_{n-1}\left\|\psi\left(u_{n-1}\right)-\mathcal{T}^{n-1} u_{n}\right\|+\alpha_{n-1}\left\|u_{n-1}-\mathcal{T}^{n-1} u_{n}\right\| \\
& +\left(1-\beta_{n-1}\right)\left[\frac{\gamma_{n-1} k_{n-1} s_{n-1}}{1-\beta_{n-1}}\left\|\mid u_{n}-u_{n-1}\right\|\right] \\
\leq & \beta_{n-1}\left\|\psi\left(u_{n-1}\right)-\mathcal{T}^{n-1} u_{n}\right\|+\alpha_{n-1}\left\|u_{n-1}-\mathcal{T}^{n-1} u_{n}\right\| \\
& +\gamma_{n-1} k_{n-1} s_{n-1}\left\|u_{n}-u_{n-1}\right\| \\
\leq & \beta_{n-1}\left\|\psi\left(u_{n-1}\right)-\mathcal{T}^{n-1} u_{n}\right\|+\alpha_{n-1}\left\|u_{n-1}-\mathcal{T}^{n-1} u_{n}\right\| \\
& +\gamma_{n-1} k_{n-1} s_{n-1}\left\|\mid u_{n}-u_{n-1}\right\|
\end{aligned}
$$

With the assumption of $\left\{\alpha_{n}\right\},\left\{\beta_{n}\right\}$ and $\lim _{n \rightarrow \infty}\left\|u_{n+1}-u_{n}\right\|=0$, we can conclude that

$$
\lim _{n \rightarrow \infty}\left\|\mathcal{T}^{n-1} u_{n}-u_{n}\right\|=0
$$

Therefore from 3.9 and 3.13, we can see from inequality 3.10, that

$$
\lim _{n \rightarrow \infty}\left\|u_{n}-\mathcal{T}\left(u_{n}\right)\right\|=0
$$

Step 4: In this step, we will show that $w_{\omega}\left(x_{n}\right) \subseteq F i x(T)$, where

$w_{\omega}\left(u_{n}\right):=\left\{u \in \mathcal{H}:\right.$ there exist a subsequence of $\left\{u_{n}\right\}$ converges weakly to $\left.u\right\}$.

Suppose that $u \in w_{w}\left(u_{n}\right)$. Then there exists a subsequence $\left\{u_{n_{i}}\right\}$ of $\left\{u_{n}\right\}$ such that $u_{n_{i}} \rightarrow x$ as $i \rightarrow \infty$. From 3.14, we have

$$
\lim _{i \rightarrow \infty}\left\|(I-\mathcal{T}) x_{n_{i}}\right\|=\lim _{n \rightarrow \infty}\left\|u_{n_{i}}-\mathcal{T} u_{n_{i}}\right\|=0
$$

. This implies that $\left\{(I-\mathcal{T}) u_{n_{i}}\right\}$ converges strongly to 0 . By using Lemma 2.2, we have $\mathcal{T} u=u$, and so $u \in \operatorname{Fix}(\mathcal{T})$.

Step 5: In this step, we will show that

$$
\lim \sup _{n \rightarrow \infty}\left\langle q-\psi(q), q-u_{n}\right\rangle \leq 0,
$$

where $q \in F(\mathcal{T})$ is the unique fixed point of $P_{F(\mathcal{T})} \circ \psi$, that is, $q=P_{F(\mathcal{T})}(\psi(z))$. Since $\left\{u_{n}\right\}$ is bounded, there exists a subsequence $\left\{u_{n_{i}}\right\}$ of $\left\{u_{n}\right\}$ such that $u_{n_{i}} \rightarrow \bar{u}$ as $i \rightarrow \infty$ for some $\bar{u} \in \mathcal{H}$ and

$$
\lim \sup _{n \rightarrow \infty}\left\langle q-\psi(q), q-u_{n}\right\rangle=\lim _{i \rightarrow \infty}\left\langle q-\psi(q), q-u_{n_{i}}\right\rangle
$$

From Step 4, we get $\bar{x} \in F(\mathcal{T})$. By using inequality 2.2, we obtain

$$
\begin{aligned}
\lim \sup _{n \rightarrow \infty}\left\langle q-\psi(q), q-u_{n}\right\rangle & =\lim _{i \rightarrow \infty}\left\langle q-\psi(q), q-u_{n_{i}}\right\rangle \\
& =\langle q-\psi(q), q-\bar{u}\rangle \leq 0
\end{aligned}
$$


Step 6: Finally, setting $\varphi_{n}=\beta_{n} q+\alpha_{n} q+\left(1-\beta_{n}\right) v_{n}$ we show that $u_{n} \rightarrow q$ as $n \rightarrow \infty$. Again, take $q \in F(\mathcal{T})$ to be the unique fixed point of the contraction $P_{F(\mathcal{T})} \circ \psi$. For each $n \in \mathbb{N}$, consider

$$
\begin{aligned}
\left\|u_{n+1}-q\right\|^{2} \leq & \left\|\varphi_{n}-q\right\|^{2}+2\left\langle u_{n+1}-\varphi_{n}, u_{n+1}-q\right\rangle \\
= & \left(1-\beta_{n}\right)^{2}\left\|v_{n}-q\right\|^{2}+2\left\langle\beta_{n}\left(\psi\left(u_{n}\right)-q\right)+\alpha_{n}\left(u_{n}-q\right), u_{n+1}-q\right\rangle \\
\leq & \left(1-\beta_{n}\right)\left\|v_{n}-q\right\|^{2}+2\left\langle\beta_{n}\left(\psi\left(u_{n}\right)-\psi(q)\right)+\beta_{n}(\psi(q)-q)+\alpha_{n}\left(u_{n}-q\right), u_{n+1}-q\right\rangle \\
\leq & \left(1-\beta_{n}\right)^{2}\left\|v_{n}-q\right\|^{2}+2 \beta_{n}\left\|\psi\left(x_{n}\right)-\psi(q)\right\|\left\|u_{n+1}-q\right\|+2 \alpha_{n}\left\|u_{n}-q\right\|\left\|u_{n+1}-q\right\| \\
& +2 \beta_{n}\left\langle\psi(q)-q, u_{n+1}-q\right\rangle \\
\leq & \left(1-\beta_{n}\right)^{2}\left\|v_{n}-q\right\|^{2}+2 \beta_{n} \alpha\left\|u_{n}-q\right\|\left\|u_{n+1}-q\right\|+2 \alpha_{n}\left\|u_{n}-q\right\|\left\|u_{n+1}-q\right\| \\
& +2 \beta_{n}\left\langle\psi(q)-q, u_{n+1}-q\right\rangle \\
\leq & \left(1-\beta_{n}\right)^{2}\left\|v_{n}-q\right\|^{2}+\left(2 \beta_{n} \alpha+2 \alpha_{n}\right)\left\|u_{n}-q\right\|\left\|u_{n+1}-q\right\| \\
& +2 \beta_{n}\left\langle\psi(q)-q, u_{n+1}-q\right\rangle
\end{aligned}
$$

For the fact that

$$
\begin{aligned}
\left\|v_{n}-q\right\|^{2}= & \left\|\frac{\gamma_{n} T^{n}\left(s_{n} u_{n}+\left(1-s_{n}\right) u_{n+1}\right)}{\left(1-\beta_{n}\right)}-q\right\|^{2} \\
\leq & \frac{\gamma_{n}^{2} s_{n}^{2} k_{n}^{2}}{\left(1-\beta_{n}\right)^{2}}\left\|u_{n}-q\right\|^{2}+\frac{\gamma_{n}^{2}\left(1-s_{n}\right)^{2} k_{n}^{2}}{\left(1-\beta_{n}\right)^{2}}\left\|u_{n+1}-q\right\|^{2} \\
& +\frac{\gamma_{n}^{2} s_{n}\left(1-s_{n}\right) k_{n}^{2}}{\left(1-\beta_{n}\right)^{2}}\left\langle u_{n}-q, u_{n+1}-q\right\rangle \\
\leq & \frac{\gamma_{n}^{2} s_{n}^{2} k_{n}^{2}}{\left(1-\beta_{n}\right)^{2}}\left\|u_{n}-q\right\|^{2}+\frac{\gamma_{n}^{2}\left(1-s_{n}\right)^{2} k_{n}^{2}}{\left(1-\beta_{n}\right)^{2}}\left\|u_{n+1}-q\right\|^{2} \\
& +\frac{\gamma_{n}^{2} s_{n}\left(1-s_{n}\right) k_{n}^{2}}{\left(1-\beta_{n}\right)^{2}}\left\|u_{n}-q\right\|\left\|u_{n+1}-q\right\| \\
& +2 \alpha_{n} \beta_{n}\left\langle\psi\left(u_{n}\right)-\psi(q), T^{n}\left(\frac{u_{n}+u_{n+1}}{2}\right)-q\right\rangle
\end{aligned}
$$

Now substituting 3.18 into 3.17 , we have the following estimation

$$
\begin{aligned}
\left\|u_{n+1}-q\right\|^{2} \leq & \gamma_{n}^{2} s_{n}^{2} k_{n}^{2}\left\|u_{n}-q\right\|^{2}+\gamma_{n}^{2}\left(1-s_{n}\right)^{2} k_{n}^{2}\left\|u_{n+1}-q\right\|^{2} \\
& +\gamma_{n}^{2} s_{n}\left(1-s_{n}\right) k_{n}^{2}\left\|u_{n}-q\right\|\left\|u_{n+1}-q\right\| \\
& +2\left(\beta_{n} \alpha+\alpha_{n}\right)\left\|u_{n}-q\right\|\left\|u_{n+1}-q\right\|+2 \beta_{n}\left\langle\psi(q)-q, u_{n+1}-q\right\rangle \\
\leq & \gamma_{n}^{2} s_{n}^{2} k_{n}^{2}\left\|u_{n}-q\right\|^{2}+\gamma_{n}^{2}\left(1-s_{n}\right)^{2} k_{n}^{2}\left\|u_{n+1}-q\right\|^{2} \\
& +\left[\gamma_{n}^{2} s_{n}\left(1-s_{n}\right) k_{n}^{2}+2\left(\beta_{n} \alpha+\alpha_{n}\right)\right]\left\|u_{n}-q\right\|\left\|u_{n+1}-q\right\| \\
& +2 \beta_{n}\left\langle\psi(q)-q, u_{n+1}-q\right\rangle
\end{aligned}
$$


Again using the fact that

$$
\begin{aligned}
\left(\left\|u_{n}-q\right\|-\left\|u_{n+1}-q\right\|\right)^{2} \leq & \left\|u_{n}-q\right\|^{2}-2\left\|u_{n}-q\right\|\left\|u_{n+1}-q\right\| \\
& +\left\|u_{n+1}-q\right\|^{2}
\end{aligned}
$$

Setting the left hand to zero, we have the following estimate

$$
\begin{aligned}
2\left\|u_{n}-q\right\|\left\|u_{n+1}-q\right\| & \leq\left\|u_{n}-q\right\|^{2}+\left\|u_{n+1}-q\right\|^{2} \\
\left\|u_{n}-q\right\|\left\|u_{n+1}-q\right\| & \leq \frac{1}{2}\left\|u_{n}-q\right\|^{2}+\frac{1}{2}\left\|u_{n+1}-q\right\|^{2}
\end{aligned}
$$

Putting inequality 3.20 in inequality 3.19 , gives the following

$$
\begin{aligned}
\left\|u_{n+1}-q\right\|^{2} \leq & \gamma_{n}^{2} s_{n}^{2} k_{n}^{2}\left\|u_{n}-q\right\|^{2}+\gamma_{n}^{2}\left(1-s_{n}\right)^{2} k_{n}^{2}\left\|u_{n+1}-q\right\|^{2} \\
& +\frac{\gamma_{n}^{2} s_{n}\left(1-s_{n}\right) k_{n}^{2}}{2}\left\|u_{n}-q\right\|^{2}+\left(\beta_{n} \alpha+\alpha_{n}\right)\left\|u_{n}-q\right\|^{2} \\
& +\frac{\gamma_{n}^{2} s_{n}\left(1-s_{n}\right) k_{n}^{2}}{2}\left\|u_{n+1}-q\right\|^{2}+\left(\beta_{n} \alpha+\alpha_{n}\right)\left\|u_{n+1}-q\right\|^{2} \\
& +2 \beta_{n}\left\langle\psi(q)-q, u_{n+1}-q\right\rangle \\
\left\|u_{n+1}-q\right\|^{2} \leq & {\left[\frac{\gamma_{n}^{2} s_{n} k_{n}^{2}\left(s_{n}+1\right)+2\left(\beta_{n} \alpha+\alpha_{n}\right)}{2}\right]\left\|u_{n}-q\right\|^{2} } \\
& +\left[\frac{\gamma_{n}^{2}\left(1-s_{n}\right)^{2} k_{n}^{2}\left(2-s_{n}\right)+2\left(\beta_{n} \alpha+\alpha_{n}\right)}{2}\right]\left\|u_{n+1}-q\right\|^{2} \\
& +2 \beta_{n}\left\langle\psi(q)-q, u_{n+1}-q\right\rangle
\end{aligned}
$$

Thus we have

$$
\begin{aligned}
(1- & {\left.\left[\frac{\gamma_{n}^{2}\left(1-s_{n}\right)^{2} k_{n}^{2}\left(2-s_{n}\right)+2\left(\beta_{n} \alpha+\alpha_{n}\right)}{2}\right]\right)\left\|u_{n+1}-q\right\|^{2} } \\
\leq & {\left[\frac{\gamma_{n}^{2} s_{n} k_{n}^{2}\left(s_{n}+1\right)+2\left(\beta_{n} \alpha+\alpha_{n}\right)}{2}\right]\left\|u_{n}-q\right\|^{2} } \\
\left\|u_{n+1}-q\right\|^{2} \leq & \frac{2 \beta_{n}\left\langle\psi(q)-q, u_{n+1}-q\right\rangle}{2-\left[\gamma_{n}^{2}\left(1-s_{n}\right)^{2} k_{n}^{2}\left(2-s_{n}\right)+2\left(\beta_{n} \alpha+\alpha_{n}\right)\right]}\left\|u_{n}-q\right\|^{2} \\
& +\frac{4 \beta_{n}}{2-\left[\gamma_{n}^{2}\left(1-s_{n}\right)^{2} k_{n}^{2}\left(2-s_{n}\right)+2\left(\beta_{n} \alpha+\alpha_{n}\right)\right]}\left\langle\psi(q)-q, u_{n+1}-q\right\rangle \\
\left\|u_{n+1}-q\right\|^{2} \leq & \left(1-\frac{2-\gamma_{n}^{2}\left(1-s_{n}\right)^{2} k_{n}^{2}\left(2-s_{n}\right)-\gamma_{n}^{2} s_{n} k_{n}^{2}\left(s_{n}+1\right)}{2-\left[\gamma_{n}^{2}\left(1-s_{n}\right)^{2} k_{n}^{2}\left(2-s_{n}\right)+2\left(\beta_{n} \alpha+\alpha_{n}\right)\right]}\left\|u_{n}-q\right\|^{2}\right. \\
& +\frac{4 \beta_{n}}{2-\left[\gamma_{n}^{2}\left(1-s_{n}\right)^{2} k_{n}^{2}\left(2-s_{n}\right)+2\left(\beta_{n} \alpha+\alpha_{n}\right)\right]}\left\langle\psi(q)-q, u_{n+1}-q\right\rangle
\end{aligned}
$$


Therefore from condition $\lim _{n \rightarrow \infty} \alpha_{n}=\lim _{n \rightarrow \infty} \beta_{n}=\lim _{n \rightarrow \infty} s_{n}=0$ in 3.1, we concludes that

$$
\begin{gathered}
\left\|u_{n+1}-q\right\|^{2} \leq\left(1-\frac{2-2 \gamma_{n}^{2} k_{n}^{2}}{2-2 \gamma_{n}^{2} k_{n}^{2}}\right)\left\|u_{n}-q\right\|^{2} \\
\lim _{n \rightarrow \infty}\left\|u_{n+1}-q\right\|^{2}=0
\end{gathered}
$$

This complete the proof.

Theorem 3.3. Let $\mathcal{M}$ be a nonempty closed convex subset a real Hilbert space $\mathcal{H}, \mathcal{T}: \mathcal{M} \rightarrow$ $\mathcal{M}$ be asymptotically nonexpansive mappings with the same sequence $\left\{k_{n}\right\} \subseteq[1, \infty)$ such that $\lim _{n \rightarrow \infty} k_{n}=1, \operatorname{Fix}(\mathcal{T}) \neq \emptyset$ and $\omega$ be a constant. Define a sequence $\left\{u_{n}\right\}$ in $\mathcal{M}$ as follows:

$$
\begin{cases}u_{1} & \in \mathcal{M} \\ u_{n+1} & =\alpha_{n} u_{n}+\beta_{n} \omega+\gamma_{n} \mathcal{T}^{n}\left(s_{n} u_{n}+\left(1-s_{n}\right) u_{n+1}\right) \forall n \in \mathbb{N}\end{cases}
$$

where $\alpha_{n}, \beta_{n}, \gamma_{n}, s_{n} \in(0,1)$ satisfying conditions $A 1-A 4$ and $\psi\left(u_{n}\right)=\omega$

$$
\lim _{n \rightarrow \infty}\left\|\mathcal{T}^{n} u_{n}-u_{n}\right\|=0
$$

Then the sequence $\left\{u_{n}\right\}$ strongly converges to a common fixed point $q$ of $\mathcal{T}$, which is also the unique solution of the following variational inequality

$$
\langle(I-\psi) u, p-u\rangle \geq 0 \quad p \in F(\mathcal{T}) .
$$

Taking $s_{n}=0$

The following corollaries holds:

Corollary 3.4. Let $\mathcal{M}$ be a nonempty closed convex subset a real Hilbert space $\mathcal{H}, \mathcal{T}: \mathcal{M} \rightarrow$ $\mathcal{M}$ be asymptotically nonexpansive mappings with the same sequence $\left\{k_{n}\right\} \subseteq[1, \infty)$ such that $\lim _{n \rightarrow \infty} k_{n}=1, \operatorname{Fix}(\mathcal{T}) \neq \emptyset$ and $\psi: \mathcal{M} \rightarrow \mathcal{M}$ be a contraction mapping with the contractive constant $\alpha \in[0,1)$. Define a sequence $\left\{u_{n}\right\}$ in $\mathcal{M}$ as follows:

$$
\begin{cases}u_{1} & \in \mathcal{M} \\ u_{n+1} & =\alpha_{n} u_{n}+\beta_{n} \psi\left(u_{n}\right)+\gamma_{n} \mathcal{T}^{n}\left(u_{n+1}\right) \forall n \in \mathbb{N}\end{cases}
$$

where $\alpha_{n}, \beta_{n}, \gamma_{n} \in(0,1)$ satisfying conditions $A 1-A 4$ without $\lim _{n \rightarrow \infty} s_{n}=0$

$$
\lim _{n \rightarrow \infty}\left\|\mathcal{T}^{n} u_{n}-u_{n}\right\|=0
$$

Then the sequence $\left\{u_{n}\right\}$ strongly converges to a common fixed point $q$ of $\mathcal{T}$, which is also the unique solution of the following variational inequality

$$
\langle(I-\psi) u, p-u\rangle \geq 0 \quad p \in F(\mathcal{T}) .
$$


Corollary 3.5. Let $\mathcal{M}$ be a nonempty closed convex subset a real Hilbert space $\mathcal{H}, \mathcal{T}: \mathcal{M} \rightarrow$ $\mathcal{M}$ be asymptotically nonexpansive mappings with the same sequence $\left\{k_{n}\right\} \subseteq[1, \infty)$ such that $\lim _{n \rightarrow \infty} k_{n}=1, \operatorname{Fix}(\mathcal{T}) \neq \emptyset$ and $u \in \mathcal{M}$ be a constant. Define a sequence $\left\{u_{n}\right\}$ in $\mathcal{M}$ as follows:

$$
\begin{cases}u_{1} & \in \mathcal{M} \\ u_{n+1} & =\alpha_{n} u_{n}+\beta_{n} \omega+\gamma_{n} \mathcal{T}^{n}\left(u_{n+1}\right) \forall n \in \mathbb{N}\end{cases}
$$

where $\alpha_{n}, \beta_{n}, \gamma_{n} \in(0,1)$ satisfying conditions $A 1-A 4$ without $\lim _{n \rightarrow \infty} s_{n}=0$

$$
\lim _{n \rightarrow \infty}\left\|\mathcal{T}^{n} u_{n}-u_{n}\right\|=0
$$

Then the sequence $\left\{u_{n}\right\}$ strongly converges to a common fixed point $q$ of $\mathcal{T}$, which is also the unique solution of the following variational inequality

$$
\langle(I-\psi) u, p-u\rangle \geq 0 \quad p \in F(\mathcal{T}) .
$$

\section{Application to convex minimization problems}

In this section, we study the problem of finding a minimizer of a convex function $\Phi$ defined from a real Hilbert space $\mathcal{M}$ to $\mathbb{R}$.

Consider the optimization problem

$$
\min _{x \in C} \Phi(x)
$$

where $\Phi: \mathcal{M} \rightarrow \mathbb{R}$ is a convex and differentiable function. Assume 4.1 is consistent, and let $\Omega \neq \emptyset$ be its set of solutions. The gradient projection algorithm generates a sequence $\left\{u_{n}\right\}$ via the iterative procedure:

$$
u_{n+1}=P_{\mathcal{M}}\left(u_{n}-\delta \nabla \Phi(u)\right)
$$

if $\nabla \Phi$ is $\theta$-inverse strongly monotone mapping and $\delta(0,2 \theta)$. The following basic results are well known.

Remark 4.1. It is well known that if $\Phi: \mathcal{M} \rightarrow \mathbb{R}$ be a real-valued differentiable convex function and $u^{*} \in \mathcal{M}$, then the point $u^{*}$ is a minimizer of $\Phi$ on $\mathcal{M}$ if and only if $d \Phi\left(u^{*}\right)=0$.

Definition 4.2. A function $\Phi: \mathcal{M} \rightarrow \mathbb{R}$ is said to be strongly convex if there exists $\alpha>0$ such that for every $u, v \in \mathcal{M}$ and $\lambda \in(0,1)$, the following inequality holds:

$$
\Phi(\lambda u+(1-\lambda) v) \leq \lambda \Phi(u)+(1-\lambda) \Phi(v)-\alpha\|u-v\|^{2} .
$$

Lemma 4.3. Let $E$ be normed linear space and $\Phi: \mathcal{M} \rightarrow \mathbb{R}$ a real-valued differentiable convex function. Assume that $\Phi$ is strongly convex. Then the differential map $d \Psi: \mathcal{M} \rightarrow \mathcal{M}$ is strongly monotone, i.e., there exists a positive constant $k$ such that

$$
\langle d \Phi(u)-d \Phi(v), u-v\rangle \geq k\|u-v\|^{2} \forall u, v \in \mathcal{M}
$$

The prove of the following theorem follows from 3.1 
Theorem 4.4. Let $\mathcal{M}$ be a nonempty closed convex subset a real Hilbert space $\mathcal{H}$. For the minimization problem 4.1, assume that $\Phi$ is (Gateaux) differentiable and the gradient $\nabla \Phi$ is a $\theta$-inverse-strongly monotone mapping for some positive real number $\theta$. Let $\psi: \mathcal{M} \rightarrow \mathcal{M}$ be a contraction with coefficient $\alpha \in[0,1)$. For a given $u_{1} \in \mathcal{M}$, let $\left\{u_{n}\right\}$ be a sequence generated by:

$$
\begin{cases}u_{1} & \in \mathcal{M} \\ u_{n+1} & =\alpha_{n} u_{n}+\beta_{n} \psi\left(u_{n}\right)+\gamma_{n} P_{\mathcal{M}}(1-\delta \nabla \Phi)\left(s_{n} u_{n}+\left(1-s_{n}\right)\left(u_{n+1}\right)\right) \forall n \in \mathbb{N}\end{cases}
$$

where $\alpha_{n}, \beta_{n}, \gamma_{n}, s_{n} \in(0,1)$ satisfying the following conditions

A1: $\alpha_{n}+\beta_{n}+\gamma_{n}=1$

A2: $\lim _{n \rightarrow \infty} \frac{k_{n}^{2}-1}{\alpha_{n}}=0$

A3: $\sum_{n=0}^{\infty} \alpha_{n}=\infty$

A4: $\lim _{n \rightarrow \infty} \gamma_{n}=1$ and $\lim _{n \rightarrow \infty} \alpha_{n}=\lim _{n \rightarrow \infty} \beta_{n}=\lim _{n \rightarrow \infty} s_{n}=0$

Then $\left\{u_{n}\right\}$ converges strongly to a solution $\left(u^{*}\right)$ of the minimization problem 4.1 , which is also the unique solution of the variational inequality

$$
\langle(I-\psi) u, p-u\rangle \geq 0 \quad p \in F(\mathcal{T}) .
$$

\section{Conflict of Interest:}

The authors declare that they have no competing interests.

\section{Availability of data and materials:}

No data were used to support this study.

\section{Funding:}

No funding was given towards this manuscript.

\section{Authors Contributions:}

All authors have contributed equally and significantly in writing this paper and also read and approved the final manuscript.

\section{Acknowledgement:}

The authors are very grateful to the editor and anonymous referees for their helpful comments.

\section{References}

[1] H. Attouch, Viscosity approximation methods for minimization problems, SIAM J. Optim. 6 (3) (1996) $769-806$. https://doi.org/10.1137/S1052623493259616.

[2] A. Moudafi, Viscosity approximation methods for fixed-points problems, J. Math. Anal. Appl. 241 (1) (2000) 46-55. https://doi.org/10.1006/jmaa.1999.6615.

[3] H.K. Xu, M.A. Alghamdi, N. Shahzad, The viscosity technique for the implicit midpoint rule of nonexpansive mappings in Hilbert spaces, Fixed Point Theory Appl. 2015 (2015) 41. https://doi.org/10.1186/s13663-015-0282-9. 
[4] Y. Ke, C. Ma, The generalized viscosity implicit rules of nonexpansive mappings in Hilbert spaces, Fixed Point Theory and Appl. 2015 (2015), 190. https://doi.org/10.1186/s13663-015-0439-6.

[5] L.C. Zhao, S.S. Chang, C.F. Wen, Viscosity approximation methods for the implicit midpoint rule of asymptotically nonexpansive mappings in Hilbert spaces, J. Nonlinear Sci. Appl. 9 (2016) 4478-4488. http: //doi .org/10 . 22436/ jnsa.009.06.86.

[6] S. He, Y. Mao, Z. Zhou, J.Q. Zhang, The generalized viscosity implicit rules of asymptotically nonexpansive mappings in Hilbert spaces, Appl. Math. Sci. 11 (12) (2017) 549-560. https://doi.org/10.12988/ams. 2017.718.

[7] J.T. Mendy, S. Rahule, Viscosity like implicit methods for zeros of monotone operators in Banach spaces, Khayyam J. Math. 2021.

[8] S.F.A. Naqvi, M.S. Khan, On the viscosity rule for common fixed points of two nonexpansive mappings in Hilbert spaces, Open J. Math. Sci. 1 (1) (2017) 111-125. http://doi.org/10.30538/oms2017.0011.

[9] J.T. Mendy, The viscosity iterative algorithms for the implicit double midpoint rule of nonexpansive mappings in Hilbert spaces, Amer. J. Math. Anal. 8 (2020), 1-8.

[10] K. Go eb el, W.A. Kirk, Topics in Me tric Fixed Point Theory, Cambridge Studies in Advanced Mathematics, vol. 28. Cambridge University Press, Cambridge (1990).

[11] H.K. Xu, Iterative algorithms for nonlinear operators, J. Lond. Math. Soc. 66 (2) (2002) 240-256. https://doi. org/10.1112/S0024610702003332.

[12] F.E. Browder, Existence of periodic solutions for nonlinear equations of evolution, Proc. Natl. Acad. Sci. USA 53 (5) (1965), 1100-1103. https://dx.doi.org/10.1073/pnas.53.5.1100.

[13] S. Dhakal, W. Sintunavarat, The viscosity implicit midpoint rule for finding common fixed points of two asymptotically nonexpansive mappings with applications, Thai J. Math. 17 (2019) 495-514.

[14] S. He , Y. Mao , Z. Zhou, J.Q. Zhang, The generalized viscosity implicit rules of asymptotically nonexpansive mappings in Hilbert Spaces, Appl. Math. Sci. 11 (2017), 549-560. https://doi.org/10.12988/ams. 2017.718. 